\title{
MODELANDO A SAZONALIDADE E O PROCESSO GERADOR DA SÉRIE DE TEMPO DO EMPREGO RURAL NO ESTADO DE SÃO PAULO ${ }^{1}$
}

Carlos Eduardo Fredo ${ }^{2}$ Mário A. Margarido

Resumo: Esse trabalho utilizou o método X-12, teste de raiz unitária sazonal e Modelo Auto-regressivo Integrado de Médias Móveis (ARIMA) para identificar e modelar o processo gerador da série temporal do emprego rural no estado de São Paulo, no período de janeiro de 1996 a dezembro de 2006. Os resultados mostram que há forte sazonalidade na demanda de mão-de-obra rural, principalmente temporária, no período de abril a agosto, em função da época da colheita nas diversas culturas. O teste de raiz unitária sazonal detectou a presença de raiz unitária sazonal, o que confirma a sazonalidade na série de emprego rural. O modelo ARIMA captou o dinamismo do emprego rural de trabalhadores temporários, pois foi necessária inclusão de parâmetros de médias móveis de pequena ordem, além de um parâmetro sazonal de média móvel e um parâmetro autoregressivo, o qual captou a contratação de trabalhadores permanentes.

Palavras-chave: Emprego rural, sazonalidade, modelo ARIMA, método X-12.

\section{Introdução}

O processo de modernização do setor agrícola brasileiro iniciou-se na década de 60, com a Revolução Verde, e teve como base o denominado modelo neoclássico, que se baseia na formulação, pelo estado, de uma política de estímulo à elevação da produtividade agrícola via choques tecnológicos, corrente contrária aos dos estruturalistas, os quais defendiam um amplo programa de reforma agrária como principal instrumento para

\footnotetext{
Recebido em: 08/09/2008; Aceito em 02/11/2008

2 Engenheiro de Computação e Pesquisador Científico de Instituto de Economia Agrícola, Água Funda, SP. E-mail: cfredo@iea.sp.gov.br.

3 Economista, Dr. em Economia Aplicada e Pesquisador do Instituto de Economia Agrícola, Água Funda, SP. E-mail: mamargarido@iea.sp.gov.br.
} 
elevar a produção e a produtividade no campo. Em linhas gerais, conforme Martins, Margarido e Bueno (2007), o

\begin{abstract}
Processo de modernização do setor agrícola no Brasil consistiu basicamente em dois pilares: oferta e demanda. O primeiro, do lado da oferta, com a concessão de estímulos para implementação de indústrias visando à produção doméstica, ou, então, com subsídios para importação de fertilizantes, defensivos, tratores, etc., além de investimentos públicos em pesquisa e difusão para utilização desses insumos modernos. Pelo lado da demanda, o principal estímulo para os produtores utilizarem esses insumos foi a constituição do programa de crédito agrícola subsidiado, o qual perdurou até meados dos anos $80^{4}$ (MARTINS, MARGARIDO e BUENO, 2007, p.31).
\end{abstract}

Os avanços tecnológicos, observados nas últimas décadas, alteraram significativamente os processos dos sistemas produtivos no setor agropecuário, em especial, as relações no mercado de trabalho. Em busca de maior produtividade, agilidade, rapidez e minimização de custos, as máquinas e equipamentos foram inseridos em operações como plantio e colheita em detrimento da força de trabalho humana, os quais, no longo prazo, se constituiriam em fortes mecanismos para expulsão da mão-deobra e reestruturação do mercado de trabalho.

Conforme Silva et al. (1983), os novos padrões tecnológicos embutidos na atividade agropecuária contribuíram consideravelmente para o aumento da produtividade, porém, como conseqüência, houve acréscimo do emprego (desemprego) sazonal. Também é necessário observar que a introdução dessas inovações tecnológicas resultou em relevantes transformações organizacionais das propriedades agropecuárias. Mais precisamente, conforme Garay (1997), a reestruturação produtiva envolve mudanças nas empresas via inovação tecnológica organizacional e de gestão, a fim de conseguir uma organização do trabalho mais integrada e flexível. No caso dos países desenvolvidos, os quais se caracterizam por

Detalhes sobre o processo de modernização do setor agrícola no Brasil podem ser encontrados em Pastore (1973), Pastore, Alves e Rizzieri (1974) e Santos (1986). 
exibir nível tecnológico mais elevado, foram adotadas leis trabalhistas mais flexíveis; além disso, o sistema educacional desses países cumpre, devidamente, sua função e consequentemente, consegue minimizar o efeito desemprego no campo.

No estado de São Paulo, várias culturas estão, quase em sua totalidade, sob trabalho mecânico, como, por exemplo, soja ou amendoim ${ }^{5}$. Outras estão em processo de mecanização, como a cana-de-açúcar, que atualmente possui 40,7\% da área cultivada (FREDO et al., 2008) já mecanizada. Nas culturas perenes, como café e laranja, a mecanização é menos acentuada, e o trabalho ainda é realizado manualmente, com eventual auxílio de equipamentos.

É inevitável que mais e mais pessoas, a cada safra agrícola, sejam desligadas de suas atividades, e pequena parcela (mediante qualificação profissional) seja absorvida em outras funções no próprio setor.

O cultivo da cana-de-açúcar, em especial, é a atividade agrícola mais importante para o estado de São Paulo, já que é responsável por 45,5\% de todo o valor da produção gerado no setor rural (TSUNECHIRO, 2008). Atividade que mais emprega mão-de-obra no campo tem sido palco para os debates sobre o mercado de trabalho.

Diferentemente de outros mercados de trabalho, o de trabalho rural é caracterizado por dois aspectos intrínsecos a esse segmento, quais sejam, o elevado nível de incerteza e a sazonalidade.

Em relação à incerteza, um evento exógeno climático, como, por exemplo, o fenômeno El Niño, pode retardar o período de chuvas e, como resultado, afetar a quantidade a ser colhida e também a demanda de mão-de-obra e fixação do nível de salários nesse setor.

Quanto à sazonalidade, apesar de seus efeitos terem declinado ao longo do tempo, em razão, principalmente, da introdução de novas tecnologias,

Especialistas apontam que os Índices de Mecanização da Soja e do Amendoim estão acima de 90\%. 
tais como processo de irrigação, o qual permite múltiplas safras ao longo do ano, eles são ainda consideráveis sobre o comportamento da produção agropecuária, alternando-se períodos de safra e entressafra para diversos produtos agropecuários. Em inúmeros casos, apesar de a tecnologia estar disponível, sua utilização pode não ser economicamente viável, em virtude de seus elevados custos de implantação, os quais, muitas vezes, não podem ser repassados para os preços ao consumidor final, em decorrência da elevada elasticidade-preço desses produtos agropecuários.

Portanto, dadas as incertezas que permeiam a produção agropecuária e a sazonalidade, o mercado de trabalho rural apresenta comportamento distinto dos demais setores econômicos. Conforme apresentado em Ray (1998), no caso do setor rural, não há um ponto de equilíbrio entre oferta e demanda de mão-de-obra, mas sim um intervalo em que ocorrerá esse equilíbrio em função da incerteza que incide sobre o processo de produção do setor agropecuário.

Para fins de formulação de políticas públicas, é importante conhecer os determinantes da demanda de mão-de-obra rural, a fim de minimizar as consequiências sociais devido à rápida introdução de novas tecnologias poupadoras de mão-de-obra. Dessa maneira, este trabalho objetiva determinar o padrão sazonal da demanda de mão-de-obra rural e seu respectivo processo gerador da série de pessoal admitido no segmento rural para o estado de São Paulo.

Além da introdução, na qual foi apresentado o referencial teórico sobre o tema, nas próximas seções serão discutidos o material e os métodos utilizados, os resultados obtidos e, por último, as considerações finais. 


\section{Material e Métodos}

\subsection{Material}

Os dados utilizados foram obtidos no Cadastro Geral de Empregados e Desempregados (CAGED), do Ministério do Trabalho e Emprego (BRASIL, 2007), disponível em CD-Rom e na Internet ${ }^{6}$. Essa base consolida, mensalmente, informações sobre número de admissões e desligamentos celetistas em todos os grandes setores econômicos brasileiros, no caso, o setor agropecuário no estado de São Paulo. Dada a obrigatoriedade de os estabelecimentos prestarem declarações sobre o fluxo de movimentação de mão-de-obra, o CAGED pode ser considerado um censo sobre o mercado de trabalho formal no Brasil. A variável escolhida foi número de admissões e desligamentos no período de janeiro de 1996 a dezembro de 2006.

\subsection{Métodos}

Foram utilizados métodos relacionados com modelos de séries de tempo, tais como método da decomposição, também denominado de método $\mathrm{X}$ 12, para obtenção dos índices sazonais para a variável número de pessoas admitidas, teste de raiz Dickey-Fuller Aumentado (ADF) e teste de raiz unitária sazonal, para tornar essa série estacionária, e modelo Autoregressivo Integrado de Médias Móveis (ARIMA), para determinar o processo gerador dessa série.

Basicamente, uma série de tempo pode ser desagregada em quatro componentes: ciclo, tendência, sazonalidade e componente aleatório. Matematicamente, uma série de tempo pode ser multiplicativa ou aditiva:

${ }_{6}$ Para ter acesso aos dados é necessário fazer parte do Programa de Disseminação das Estatísticas do Trabalho (PDET). 


$$
\hat{Y} m_{t}=\hat{T}_{t} * \hat{S}_{t} * \hat{C}_{t} * \hat{I}_{t}
$$

$\mathrm{ou}$

$$
\hat{Y} a_{t}=\hat{T}_{t}+\hat{S}_{t}+\hat{C}_{t}+\hat{I}_{t}
$$

em que $\hat{Y} m_{t}$ é série de tempo classificada como multiplicativa; $\hat{Y} a_{t}$, série de tempo classificada como aditiva; $\hat{T}_{t}$, tendência; $\hat{S}_{t}$, sazonalidade; $\hat{C}_{t}$, ciclo; e $\hat{I}_{t}$, componente aleatório.

A tendência capta elementos de longo prazo relacionados com a série de tempo; pode ser determinística, ou seja, pode ser uma função matemática, ou então estocástica ${ }^{7}$, isto é, resultado de um processo aleatório.

O componente sazonal capta os padrões regulares da série de tempo, tais como mudanças de temperatura, índice pluviométrico, safra ou entressafra de produtos agropecuários, vendas da indústria, vendas do varejo, entre outros.

Os ciclos são caracterizados por longas ondas, mais ou menos regulares, em torno de uma linha de tendência. O interesse pelos ciclos está diretamente relacionado com seus pontos de mudanças, também denominados de pontos de inflexão ${ }^{8}$, duração, freqüência, etc.

Detalhes sobre o método X-12 podem ser encontrados em Yaffee e McGee (2000). No cálculo do Coeficiente de Amplitude (CA) foi utilizado o trabalho de Freitas et al. (1998).

\footnotetext{
Neste caso, a tendência estocástica, em economia, mostra alterações no nível da série de tempo, as quais podem ser resultado de mudanças sociais, tecnológicas, condições de mercado, meio ambiente, entre outros.

8 Em economia, o interesse em conhecer adequadamente este componente da série de tempo está relacionado com os ciclos econômicos, visando determinar períodos de prosperidade, recessão, entre outros.
} 
O pacote econométrico utilizado na obtenção dos índices sazonais foi o Statistical Analysis Software (SAS) versão 9.1, conforme descrito em U.S. Bureau of the Census (1999).

Para testar a presença de raiz unitária na série foram utilizados dois tipos de testes de raiz unitária. A determinação da correta ordem de integração das variáveis tem expressiva relevância para aqueles que transitam pelo campo dos modelos de séries temporais. De acordo com Rao (1994), os "métodos padrões de estimação, que são rotineiramente utilizados em trabalhos de aplicação econométrica, têm como base o pressuposto de que a média e a variância das variáveis são constantes e independentes do tempo. Contudo, a aplicação dos testes de raiz unitária tem mostrado que esses pressupostos não são válidos para um grande número de séries de tempo macroeconômicas ${ }^{9}$. Variáveis cujas médias e variâncias mudam ao longo do tempo são denominadas não - estacionárias ou variáveis com raiz unitária” (RAO, 1994, p.2). Ainda segundo Rao (1994), a utilização de métodos tradicionais para estimar o relacionamento entre variáveis que tenham raiz unitária, tais como o método de mínimos quadrados ordinários, fornece inferências viesadas. Mais precisamente, dado que, diante da presença de raiz unitária, as médias e variâncias das variáveis não são invariantes ao longo do tempo, as estatísticas obtidas a partir desses métodos tradicionais falham em convergir para seus respectivos valores verdadeiros, quando se amplia o tamanho da amostra. Como resultado, obtém-se relacionamento espúrio entre as variáveis, conforme apresentado em Granger e Newbold (1974) e Phillips (1996). Assim, a determinação da ordem de integração das variáveis torna-se imprescindível.

Uma série de tempo será estacionária quando preencher três condições. Conforme Freitas, Margarido, Barbosa e Franca (2001), um “processo estocástico será dito estacionário, ou mais especificamente terá fraca

\footnotetext{
"Isto porque se a hipótese de raiz unitária for verdadeira para uma série, os choques aleatórios que ela sofresse gerariam na mesma um efeito permanente. As flutuações não seriam transitórias, derrubando, por exemplo, as teorias de que os ciclos econômicos seriam flutuações temporárias em torno de uma tendência" (ALENCAR, 1998, p. 171). O trabalho utilizado como referencial para o fato de que a maioria das séries econômicas possuem raiz unitária é o de Nelson e Plosser (1982).
} 
estacionariedade, quando preencher três requisitos básicos. Em primeiro lugar, sua média terá de ser constante ao longo do tempo (E(y) é constante para todo $t$ ). Outra condição é que a sua variância também seja invariante ao longo do tempo $(\operatorname{Var}(\mathrm{y})$ é constante para todo $t)$. Finalmente, a sua covariância indicará que a autocorrelação entre dois valores de $y$, tomados a partir de dois períodos de tempo distintos, dependerá somente do intervalo do tempo entre esses dois valores, e não da sua data $\left(\operatorname{Cov}\left(\mathrm{y}_{\mathrm{t}}, \mathrm{y}_{\mathrm{t}+\mathrm{s}}\right), \mathrm{e}\right.$ será constante para todo $t$ que não seja igual a $s$ " (FREITAS, MARGARIDO, BARBOSA, FRANCA, 2001, p.4).

Para identificar a ordem de integração das variáveis, geralmente se utiliza o teste de raiz unitária convencional do tipo Dickey-Fuller Aumentado (DICKEY e FULLER, 1979 e 1981). Na determinação do número de defasagens utiliza-se o menor valor de algum critério de informação, como, por exemplo, os Critérios de Schwarz (SBC) $)^{10}$ ou Akaike (AIC) ${ }^{11}$, entre outros, com vistas em eliminar a autocorrelação dos resíduos.

$\mathrm{O}$ teste de raiz unitária $\mathrm{ADF}^{12}$ testa a hipótese nula de presença de raiz unitária ( $\rho=1$ ),contra a hipótese alternativa de que a série é estacionária $(\rho<1)$, considerando a presença ou não da constante e, ou, da tendência (denominadas de testes $\tau, \tau_{\mu}, \tau_{{ }}$). Os valores críticos para os testes individuais encontram-se em Mackinnon (1991), enquanto os valores tabelados para os testes conjuntos encontram-se em Dickey e Fuller (1981).

A sazonalidade é um aspecto relevante para explicar determinadas séries econômicas. Os testes de raiz unitária convencionais não podem ser utilizados quando a série econômica apresentar padrão sazonal, dado que somente a aplicação de uma diferença de ordem um não é suficiente para tornar a série estacionária.

\footnotetext{
${ }_{10}$ Schwarz (1978).

${ }^{11}$ Akaike (1974).

12 Maiores detalhes sobre o procedimento operacional passo a passo, para execução dos testes de raiz unitária dos tipos Dickey-Fuller (DF), Dickey-Fuller Aumentado (ADF) e o teste não - paramétrico Phillips-Perron (PP), podem ser encontrados em Margarido e Anefalos (1999).
} 
Dickey, Hasza e Fuller (1984) desenvolveram teste para detectar presença de raiz unitária sazonal, o qual se baseia no teste Dickey-Fuller Aumentado (ADF). Nesse caso, a ordem da diferença é denominada de diferença sazonal e vai depender de sua regularidade. Para séries mensais com sazonalidade anual, a diferença é de ordem $1(d=12)$; para séries mensais com padrão semestral, de ordem $2(d=6)$; para séries mensais com padrão trimestral, de ordem $4(d=4)$.

O teste Dickey-Hasza-Fuller (DHF) tem como base a seguinte autoregressão:

$$
y_{t}=\sum_{s=1}^{4} \mu_{s} D_{s t}+\beta t+\rho_{4} y_{t-4}+u_{t}
$$

e que $D_{s t}$ são variáveis binárias (dummies) sazonais; $t$, tendência determinística. A hipótese nula $\left(H_{0}\right)$ é que $\rho_{4}=1$.

Outra forma de escrever esse modelo é a seguinte:

$$
\nabla^{4} y_{t}=\alpha y_{t-4}+d_{1} S_{1 t}+d_{2} S_{2 t}+d_{3} S_{3 t}+d_{4} S_{4 t}+\beta t+\sum_{i=1}^{k} \phi_{i} \nabla^{4} y_{t-i}+u_{t},
$$

em que $S_{i t}(i=1,2,3,4)$ são variáveis dummies sazonais; $t$, tendência determinística, enquanto $\nabla^{4} y_{t}=y_{t}-y_{t-4}$ é a diferença sazonal. A hipótese nula é que $\alpha=0$. Finalmente, o termo $\sum_{i=1}^{k} \phi_{i} \nabla^{4} y_{t-i}$, o qual representa a variável diferenciada e defasada, é utilizado para eliminar a autocorrelação dos resíduos. O número de defasagens pode ser determinado pela utilização de algum critério de informação AIC ou SBC, ou então pelo critério apresentado em Ng e Perron (1995). 
Os modelos Auto-regressivos Integrados de Médias Móveis (ARIMA) têm como base o trabalho de Box, Jenkins e Reinsel (1994). De acordo com esse método, uma série temporal pode ser explicada, em parte, por ela mesma ${ }^{13}$, tendo como base seus respectivos valores passados, também denominados de parâmetros auto-regressivos (AR) e, ou, pelos próprios erros presente e passado (parâmetros de médias móveis (MA)). Basicamente, o modelo ARIMA tem a seguinte representação:

$$
\tilde{y}_{t}=\frac{\theta() B \Theta\left(B^{s}\right)}{\phi(B) \Phi\left(B^{s}\right)} a_{t}
$$

em que $\widetilde{\mathrm{y}}_{\mathrm{t}}$ é a variável $y_{t}$ diferenciada ${ }^{14}$ e centrada em relação à sua própria média, enquanto $\theta(B)$ e $\phi(B)$ são polinômios que representam o operador de média móvel de ordem $q$ e o operador auto-regressivo de ordem $p$, respectivamente, e $\Theta\left(B^{s}\right)$ e $\Phi\left(B^{s}\right)$ representam os polinômios de médias móveis sazonal e auto-regressivo sazonal, respectivamente ${ }^{15}$. A ordem de integração de uma variável é o número de vezes que essa variável deve ser diferenciada para tornar-se estacionária.

Para verificar a efetividade da filtragem será utilizado o teste Ljung-Box (1978), o qual é dado pela seguinte fórmula:

$$
Q=T(T+2) \sum_{k=1}^{s} r_{k}^{2} /(T-k),
$$

em que $T$ é número de observações; $r_{k}$, autocorrelação na defasagem $k$; $Q$ tem distribuição $\chi^{2}$ com $k$ graus de liberdade. A série será ruído

\footnotetext{
13 O modelo ARIMA também é denominado de univariado.

14 No caso em que a variável necessite ser diferenciada para se tornar estacionária, essa diferença pode ser somente regular, somente sazonal ou, então, ambas conjuntamente.

15 Maiores detalhes sobre os modelos ARIMAs podem ser encontrados em Margarido (1998), Box; Jenkins; Reinsel (1994), Mills (1990) e Vandaele (1983), entre outros.
} 
branco quando $Q$ tiver valor igual a zero, ou seja, não houver presença de autocorrelação nos resíduos da série. O teste Ljung-Box é superior ao tradicional teste de Box-Pierce ${ }^{16}$, pois possui performance superior para casos com pequenas amostras em relação ao teste Box-Pierce.

\section{Análise de Resultados}

Em função das características inerentes ao próprio ciclo de produção da agropecuária, a demanda de mão-de-obra, no segmento rural, concentrase em determinados períodos do ano. O menor nível de emprego tende a concentrar-se entre os meses de outubro a março, período em que se inicia a entressafra e, como conseqüência, a redução na demanda de mão-de-obra no campo. Esse período corresponde ao plantio da canade-açúcar e ao início das safras de feijão e amendoim das águas (BAPTISTELLA et al; 1994). O mês com menor demanda de mão-deobra no campo é dezembro, e seu índice sazonal é igual a 40,749. O maior volume de pessoas empregadas no campo tende a ocorrer de abril a setembro (período de safra), enquanto o pico de mão-de-obra no campo ocorre em maio e corresponde a um índice sazonal igual a 167,61 (Figura 1). Conforme Baptistella et al. (1994), no início da década de 90 já havia indícios de antecipação da colheita da cana-de-açúcar para maio, o que confirma o resultado apresentado.

Há de se considerar, na série de dados, a forte influência da cana-deaçúcar, que hoje é a atividade agropecuária que mais emprega no setor rural, principalmente no período da colheita, e que ainda está em processo de mecanização.

O coeficiente de amplitude é igual a 121,7708, enquanto a média de todos os índices sazonais é, aproximadamente, igual a $100(99,99708)$. Esse resultado mostra que há expressiva variação na demanda de mãode-obra rural ao longo do ano, em torno de $21,77 \%$. Apesar das inovações

16 Box e Pierce (1970). 
tecnológicas introduzidas no meio rural, como, por exemplo, a irrigação, ainda assim a questão da sazonalidade da produção agrícola continua a exercer forte pressão na condução do processo de produção agropecuária. Também foram calculadas as médias dos índices sazonais do período de entressafra $(74,4675)$ e safra $(125,5267)$, conforme pode ser visualizado na Figura 2.

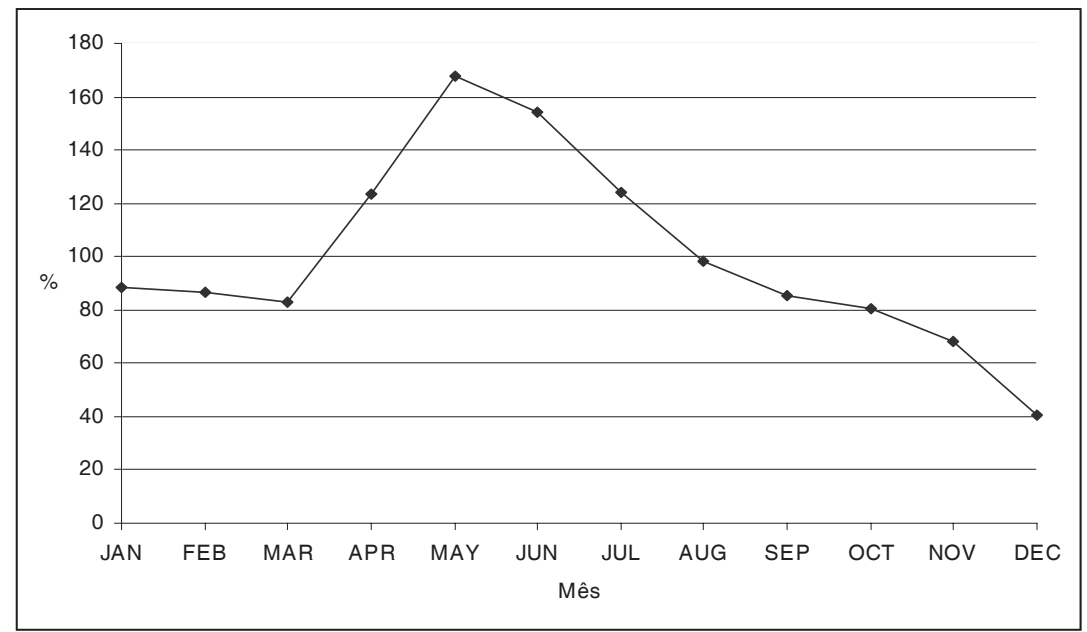

Figura 1 - Índices sazonais, pessoal admitido, 1996-2006.

Fonte: Elaborada pelos autores a partir de dados básicos do CAGED. 


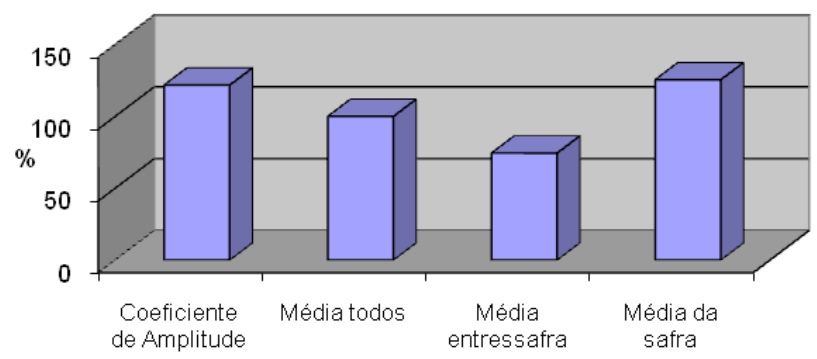

Figura 2 - Coeficiente de amplitude, média de todos índices sazonais, média dos índices sazonais da entressafra e média dos índices sazonais da safra.

Fonte: Elaborada pelos autores a partir de dados básicos do CAGED.

O primeiro passo consistiu na identificação do modelo ARIMA com a variável número de pessoas admitidas em nível ${ }^{17}$. A visualização do respectivo correlograma mostrou que o processo dessa variável tem memória longa, ou seja, decai lentamente ao longo do tempo, o que é um indicativo da presença de raiz unitária. Outro aspecto observado foi que o correlograma apresenta "picos" nas defasagens 12 e 24, o que indica a presença de sazonalidade nessa série.

Em razão das constatações de que a respectiva série em nível não é estacionária e que possui sazonalidade, foi realizado o teste de raiz unitária sazonal proposto por Dickey, Hasza e Fuller (1984), o qual permite testar a presença de raiz unitária sazonal pelos dois modelos. O primeiro é o modelo que não utiliza parâmetro relacionado com tendência determinística, porém utiliza uma constante (ou intercepto). Nesse caso, a estatística utilizada é denominada de $\tau_{\mu}$. O segundo modelo não utiliza

17 Nesse ponto, é necessário realçar que está sendo utilizado o logaritmo da variável pessoal admitido em todos os estágios relacionados com o modelo ARIMA. 
nem tendência determinística, nem constante, e utiliza a estatística $\tau$. Em ambos os testes, a hipótese nula é de que há raiz unitária sazonal, enquanto na hipótese alternativa pressupõe-se que não haja raiz unitária sazonal.

No caso do modelo somente com constante, a hipótese nula pode ser rejeitada, pois há somente $2,51 \%$ de probabilidade de rejeitar essa hipótese e ela ser verdadeira. $\mathrm{O}$ mesmo resultado não se aplica ao modelo sem constante e sem tendência, dado que há $99,99 \%$ de probabilidade de se cometer o Erro Tipo I, ou seja, há 99,99\% de probabilidade de se rejeitar a hipótese nula de que há raiz unitária sazonal e de essa hipótese ser verdadeira. Portanto, apesar desse conflito entre os resultados dos testes de raiz unitária sazonal, ainda assim pode-se considerar que essa série tenha raiz unitária sazonal, dado que a visualização do correlograma da função de autocorrelação da variável em nível indica forte presença do componente sazonal (Figura 3). Como resultado, pode-se inferir que essa série possua raiz unitária sazonal (Tabela 1). 


\section{Autocorrelations}

Lag Covariance Correlation -198765432101234567891 Std Error

\begin{tabular}{|c|c|c|c|c|}
\hline 0 & 0.227694 & 1.00000 & & $|* * * * * * * * * * * * * * * * * * * *|$ \\
\hline 1 & 0.170637 & 0.74941 & & $|* * * * * * * * * * * * * * * * \quad| 0.087039$ \\
\hline 2 & 0.125889 & 0.55288 & & $\mid * * * * * * * * * * *$ \\
\hline 3 & 0.092793 & 0.40753 & & |********* \\
\hline 4 & 0.047022 & 0.20651 & & 0.152424 \\
\hline 5 & 0.0026797 & 0.01177 & & 0.154529 \\
\hline 6 & -0.011052 & -.04854 & & 0.154536 \\
\hline 7 & 0.0020293 & 0.00891 & & 0.154651 \\
\hline 8 & 0.046395 & 0.20376 & . & 0.154655 \\
\hline 9 & 0.086030 & 0.37783 & & |********** \\
\hline 10 & 0.111519 & 0.48977 & & \begin{tabular}{l|l}
$\mid * * * * * * * * * *$ & 0.163433
\end{tabular} \\
\hline 11 & 0.145435 & 0.63873 & . & $\mid$\begin{tabular}{|l|l}
$\mid * * * * * * * * * * * * *$ & 0.174198
\end{tabular} \\
\hline 12 & 0.187085 & 0.82165 & & $|* * * * * * * * * * * * * * * *|$ \\
\hline 13 & 0.140469 & 0.61692 & . & |************ \\
\hline 14 & 0.102029 & 0.44810 & . & 0.229176 \\
\hline 15 & 0.073095 & 0.32102 & . & 0.235720 \\
\hline 16 & 0.028618 & 0.12568 & 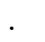 & 0.239009 \\
\hline 17 & -0.013269 & -.05828 & - & 0.239510 \\
\hline 18 & -0.026799 & -.11770 & 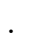 & 0.239617 \\
\hline 19 & -0.017990 & -.07901 & . & 0.240055 \\
\hline 20 & 0.023583 & 0.10357 & . & 0.240251 \\
\hline 21 & 0.060055 & 0.26375 & . & 0.240589 \\
\hline 22 & 0.083166 & 0.36525 & . & 0.242770 \\
\hline 23 & 0.109883 & 0.48259 & . & |*********** \\
\hline 24 & 0.143680 & 0.63102 & & *************** \\
\hline
\end{tabular}

Figura 3. - Função de Autocorrelação da variável Número de Pessoas Admitidas em nível.

Fonte: Elaborada pelos autores a partir de dados básicos do CAGED. 
Tabela 1. - Resultados dos testes de raiz unitária sazonais variável em nível

\begin{tabular}{lll}
\hline Modelo & Estatística $\tau$ & $\operatorname{Pr}<\tau$ \\
\hline Sem tendência e sem constante $(\tau)$ & 4.40 & 0.9999 \\
Sem tendência, porém, com constante $\left(\tau_{\mu}\right)$ & -2.35 & 0.0251 \\
\hline
\end{tabular}

Fonte: Elaborada pelos autores a partir de dados básicos do CAGED.

Em virtude dos resultados dos testes de raiz unitária com a variável em nível, para eliminar esse comportamento sazonal aplicou-se uma diferença de ordem 12 e efetuaram-se, novamente, os testes de raiz unitária sazonal. Em ambos os modelos verifica-se que a hipótese de raiz unitária sazonal pode ser rejeitada, uma vez que, nos dois casos, a probabilidade de se cometer o Erro do Tipo I, isto é, rejeitar a hipótese nula e de ela ser verdadeira, está abaixo do nível de significância de 1,0\%. Portanto, essa série não apresenta mais o problema relacionado com a presença de raiz unitária sazonal (Tabela 2).

Tabela 2. - Resultados dos testes de raiz unitária sazonal variável diferenciada de ordem 12

\begin{tabular}{lll}
\hline Modelo & Estatística $\tau$ & $\operatorname{Pr}<\tau$ \\
\hline Sem tendência e sem constante $(\tau)$ & -10.35 & $<.0001$ \\
Sem tendência, porém com constante $\left(\tau_{\mu}\right)$ & -13.38 & $<.0001$ \\
\hline
\end{tabular}

Fonte: Elaborada pelos autores a partir de dados básicos do CAGED.

Apesar de a raiz unitária sazonal ter sido eliminada pela aplicação de uma diferença de ordem 12, ainda assim a série apresenta raiz unitária, conforme pôde ser visualizado no respectivo correlograma, pois decai lentamente ao longo do tempo. Assim, foi realizado o teste de raiz unitária ADF, porém, antes da execução desse teste, foi determinado o seu número de defasagens utilizando o critério de informação de Schwarz (1978). O 
resultado do critério de Schwarz indica a necessidade de quatro defasagens para eliminar a autocorrelação dos resíduos no teste ADF.

Os resultados dos testes de raiz unitária mostram que a hipótese nula de raiz unitária pode ser rejeitada para os modelos com tendência e constante e para o modelo somente com constante. No entanto, a hipótese nula de raiz unitária não pode ser rejeitada, pois há 56,0\% de probabilidade de se rejeitar a hipótese nula e de ela ser rejeitada (Tabela 3).

Tabela 3. Resultados do teste de raiz unitária Dickey-Fuller Aumentado (ADF), variável em nível

\begin{tabular}{lllllll}
\hline Variável & $\tau_{\tau}{ }^{1}$ & $\operatorname{Pr}<\tau_{\tau}$ & $\tau_{\mu}{ }^{2}$ & $\operatorname{Pr}<\tau_{\mu}$ & $\tau^{3}$ & $\operatorname{Pr}<\tau$ \\
\hline LAMDT & $-9.34^{*}$ & $<.0001$ & $-4.87^{*}$ & 0.0001 & -0.34 & 0.5600 \\
\hline
\end{tabular}

${ }^{1}$ Modelo com tendência e constante; ${ }^{2}$ Modelo sem tendência, porém com constante; ${ }^{3}$ Modelo sem tendência e sem constante. * Significativo a 1,0\%.

Fonte: Elaborada pelos autores a partir de dados básicos do CAGED.

Diante desses conflitos em relação aos resultados dos testes ADF, utilizouse a visualização do respectivo correlograma para a tomada de decisão sobre a ordem de integração da variável, o qual mostrou que a série decai lentamente, indicando presença de raiz unitária. Assim, o teste de raiz unitária foi realizado novamente, porém, dessa vez, com a variável diferenciada de ordem um.

Os resultados dos testes de raiz unitária para a variável diferenciada mostram que, em todos os três casos, a hipótese nula de raiz unitária pode ser rejeitada, ou seja, não se rejeita a hipótese nula de que a variável seja estacionária, quando diferenciada (Tabela 4). 
Tabela 4. Resultados do Teste de Raiz Unitária Dickey-Fuller Aumentado (ADF), variável diferenciada

\begin{tabular}{lllllll}
\hline Variável & $\tau_{\tau}{ }^{1}$ & $\operatorname{Pr}<\tau_{\tau}$ & $\tau_{\mu}{ }^{2}$ & $\operatorname{Pr}<\tau_{\mu}$ & $\tau^{3}$ & $\operatorname{Pr}<\tau$ \\
\hline LAMDT & $-10.30^{*}$ & $<.0001$ & $-10.35^{*}$ & 0.0001 & $-10.39^{*}$ & 0.0001 \\
\hline${ }^{1}$ Modelo com tendência e constante; ${ }^{2}$ Modelo sem tendência, porém com \\
constante; ${ }^{3}$ Modelo sem tendência e sem constante. ${ }^{*}$ Significativo a $1,0 \%$. \\
Fonte: Elaborada pelos autores a partir de dados básicos do CAGED.
\end{tabular}

Determinada a ordem de integração da variável, o próximo passo consistiu na estimação do modelo ARIMA.

A elaboração do modelo ARIMA, para tornar a série ruído branco (white noise), exigiu além de duas diferenças, sendo uma de ordem um e outra de ordem 12, mais quatro parâmetros, sendo dois de médias móveis, um de média móvel sazonal e um auto-regressivo.

A estimativa do parâmetro de médias móveis de ordem um é igual a 0,50709. Isso quer dizer que, na média, os erros da variável pessoal admitido são corrigidos em torno de $50,71 \%$ no período atual, em relação a seu próprio valor do mês anterior. O mesmo tipo de análise vale para o parâmetro de médias móveis, de ordem 2. Nesse caso, na média, o desequilíbrio no número de pessoal admitido é corrigido em torno de $24,18 \%$ no período atual, tendo como base seu respectivo valor passado de dois meses. Aparentemente, esses dois parâmetros de médias móveis, de pequena ordem, captam o dinamismo (rotatividade) do mercado de trabalho por mão-de-obra rural, no curto prazo (Tabela 5).

Dadas as características inerentes da produção agrícola, em que há alternância de períodos de safra e entressafra, isso induz a um comportamento expressivamente sazonal da demanda de mão-de-obra. Apesar da necessidade de se aplicar uma diferença sazonal para tornar a série estacionária, ainda assim esse procedimento não foi suficiente para remover toda sazonalidade da série, pois foi necessária a inclusão de um parâmetro sazonal de ordem um. O valor para a estimativa desse 
parâmetro sazonal mostra que, na média, os erros da variável pessoal admitido são corrigidos em 78,33\%, a cada 12 meses (Tabela 5).

Finalmente, houve necessidade de adicionar um parâmetro autoregressivo, cujo valor da estimativa corresponde a 0,2390. Isso quer dizer que o número de pessoas admitidas no período atual $(t)$ é influenciado pelo número de pessoas admitidas há sete meses atrás $(t-7)$ em cerca de 23,91\% (Tabela 5).

Tabela 5. - Resultados dos parâmetros estimados para o Modelo ARIMA, pessoal admitido, janeiro de 1996 a dezembro de 2006

\begin{tabular}{llllll}
\hline Parâmetro & Estimativa & Desvio-Padrão & $\mathrm{T}$ & $\operatorname{Pr}>|\mathrm{t}|$ & Defasagem \\
\hline MA (1) & 0.50709 & 0.09058 & $5.60 *$ & $<.0001$ & 1 \\
MA (2) & 0.24183 & 0.09179 & $2.63 *$ & 0.0096 & 2 \\
MA (12) & 0.78331 & 0.07051 & $11.11^{*}$ & $<.0001$ & 12 \\
AR (7) & 0.23906 & 0.09279 & $2.58 * *$ & 0.0112 & 7 \\
\hline
\end{tabular}

*Significativo a $1,0 \%$, **Significativo a $5,0 \%$.

Fonte: Elaborada pelos autores a partir de dados básicos do CAGED.

Antes de entender este parâmetro, é necessário ter em mente que, basicamente, há dois tipos de contratos de trabalho no setor agropecuário. O primeiro envolve trabalhadores que são contratados para execução de tarefas no curto prazo, como, por exemplo, somente no período de colheita. É este grupo que engrossa o volume de contratações que se realizam no ano e concentram em cinco meses (abril a agosto). Conforme Ray (1998), os trabalhadores contratados pelo regime temporal de curto prazo, geralmente, são alocados em tarefas onde a supervisão de suas respectivas funções é relativamente fácil de acompanhar, tais como colheita e semeadura; consequentemente, seu desligamento do processo produtivo é mais simples em relação ao dos empregados permanentes.

A Tabela 6 exemplifica o curto período de tempo em que esses trabalhadores permanecem em suas funções. Do total de demissões realizadas no ano de 2006 no setor, $45,6 \%$ permaneceram até seis meses 
nas atividades agropecuárias, enquanto $80 \%$ não permaneceram um ano inteiro no setor.

Tabela 6 - Distribuição de desligamentos por faixa de tempo de permanência, setor agropecuário, estado de São Paulo, 2006.

\begin{tabular}{lll}
\hline Meses & Número de demissões & $\%$ de demissões \\
\hline De 1,0 a 2,9 meses & 80.063 & 22,8 \\
De 3,0 a 5,9 meses & 80.278 & 22,8 \\
De 6,0 a 11,9 meses & 122.528 & 34,9 \\
De 12,0 a 35,9 meses & 35.103 & 10,0 \\
Mais de 36,0 meses & 21.040 & 6,0 \\
Ignorado* & 12.558 & 3,6 \\
Total & 351.570 & 100,0 \\
\hline
\end{tabular}

* O atributo "Ignorado" refere-se aos postos de trabalho sem informação (ou informação duvidosa) quanto ao tempo de permanência no emprego.

Fonte: CAGED, MTE.

O segundo tipo é composto por trabalhadores com contratos de longo prazo (trabalhadores permanentes) e alocados em tarefas mais específicas, ou seja, com certa especialização, cujas respectivas funções são mais complexas de se monitorar, tais como aplicações de fertilizantes, defensivos, utilização de irrigação, etc. No entanto, esses empregados podem, eventualmente, participar do processo de colheita, a exemplo do cultivo do café, no qual uma colheita seletiva significa um produto com maior qualidade e maior aceitação no mercado.

Quando a demanda de trabalhadores temporários diminui a partir de setembro até março, a principal movimentação do trabalho é por parte dos permanentes, destacando-se, portanto, um período que compreende sete meses do ano, o que comprova o parâmetro auto-regressivo de ordem 7 . 
Matematicamente, esse modelo ARIMA é escrito da seguinte forma:

$$
\nabla_{s=1}^{12} \nabla \operatorname{ladm}_{t}=\frac{\left(1-0,5070 B-0,2418 B^{2}\right)\left(1-0,7833 B^{12}\right) a_{t}}{(1-0,2390 B)}
$$

Compactamente, esse modelo univariado é escrito como sendo um modelo $\operatorname{ARIMA}(7,1,2)(0,1,1)_{12}$.

Após a identificação e a estimação dos parâmetros, a última fase para análise do modelo ARIMA é a sua verificação, isto é, conferir se o processo de filtragem da variável foi efetivo, de modo a tornar os resíduos não - correlacionados, também denominados de ruído branco (white noise). Para verificar se os resíduos são não - correlacionados, utilizouse o teste Ljung-Box, cuja hipótese nula é de que o grupo de autocorrelações é significativamente diferente de zero. Conforme os resultados obtidos, a hipótese nula de que os resíduos sejam diferentes de zero não poderá ser rejeitada e nenhuma das 24 defasagens, conforme os respectivos valores das probabilidades. Isso confirma a efetividade filtragem, de acordo com a Tabela 5.

Tabela 5. - Resultados do teste Ljung-Box, pessoal admitido, janeiro de 1996 a dezembro de 2006

\begin{tabular}{llllllllll}
\hline Defasagem & $\chi^{2}$ & $\begin{array}{l}\text { Graus de } \\
\text { Liberdade }\end{array}$ & $\begin{array}{c}P \text {-value } \\
\chi^{2}\end{array}$ & \multicolumn{2}{l}{ Autocorrelações } & & & & \\
\hline 6 & 0,32 & 2 & 0,8534 & 0,005 & $-0,011$ & 0,008 & 0,033 & 0,031 & 0,017 \\
12 & 3,36 & 8 & 0,9101 & 0,012 & 0,104 & $-0,053$ & $-0,068$ & 0,030 & 0,062 \\
18 & 12,99 & 14 & 0,5272 & 0,133 & $-0,016$ & 0,203 & $-0,096$ & $-0,025$ & $-0,026$ \\
24 & 20,77 & 20 & 0,4111 & $-0,085$ & $-0,019$ & $-0,137$ & 0,051 & 0,107 & $-0,110$ \\
\hline
\end{tabular}

Fonte: Elaborada pelos autores a partir de dados básicos do CAGED.

A forte sazonalidade observada no mercado de trabalho rural, com altos picos de admissões no período da colheita, sofrerá mudanças num futuro próximo, por diversas razões. Uma delas é o alto custo de se ter um trabalhador temporário por apenas alguns meses. A fiscalização das 
delegacias regionais do trabalho tem pressionado os produtores, a cada safra, para que formalizem o registro na carteira desses trabalhadores. Conforme Rezende (2006), no empregador recai uma série de encargos sociais decorrentes da Consolidação das Leis do Trabalho (CLT); portanto, para ter esse trabalhador com carteira assinada, com vistas em minimizar o custo, é preferível a substituição de oitenta trabalhadores por uma máquina e por um tratorista permanente (estimativa para o cultivo de cana) que, diga-se de passagem, é mais qualificado que os demais.

Dessa maneira, se a mecanização é uma solução atrativa, do ponto de vista do proprietário rural (agilidade, rapidez e aumento da produtividade), ela se torna mais acelerada com a Lei no. 11.241/2002, cuja finalidade principal é mitigar a emissão de gases de efeito estufa, proibindo gradativamente a queima da cana até 2021, em áreas possíveis de serem mecanizadas, e até 2031, para o restante das áreas cultivadas.

Embora o artigo Dez, da Lei 11.241, prevê a criação de mecanismos para realocação da mão-de-obra ou que o impacto gerado pela mecanização não seja propulsor de desemprego, pouco se tem feito para diminuir os impactos sociais desse processo.

Mais recentemente, em 2007/08, surgiram os Protocolos Agroambientais estabelecidos entre governo paulista, usinas e fornecedores, o que antecipou ainda mais o cronograma (2014 para áreas mecanizáveis e 2017 para não-mecanizáveis).

É um acordo de intenções, na qual aqueles que aderirem ao Protocolo e cumprirem suas exigências serão beneficiados com o selo agroambiental, que facilitará a comercialização do etanol (Secretaria do Meio Ambiente (SMA), 2007). Os Protocolos, portanto, aceleram ainda mais o processo irreversível da mecanização.

Nada impede o uso do corte manual sobre a cana crua (evitando a queima), porém haverá maior desgaste físico, serão piores as condições de trabalho e cairá a produtividade desses trabalhadores, encarecendo ainda mais os custos ao produtor e inviabilizando, portanto, essa alternativa. 


\section{Considerações finais}

Quando se comparam os resultados obtidos neste trabalho com outro estudo realizado anteriormente por Bapistella et al. (1994), ainda não se observa tendência de distribuição mais equilibrada de contratações no emprego da mão-de-obra ao longo do ano. A sazonalidade ainda é persistente, vistos que os picos de contratação no mês de julho coincidem com a colheita das culturas anuais e há diminuição das contratações na entressafra. Faz-se necessário um aprofundamento do estudo, isolando a série aqui utilizada em duas: uma exclusiva para a cultura da cana-deaçúcar e outra para as demais culturas. Dessa maneira, dada a acelerada mecanização na colheita da cana, será possível detectar se há reestruturação nas contratações da mão-de-obra. Em contrapartida, para as demais culturas seria possível observar a possibilidade de alteração nos contratos de trabalho, ou seja, diminuição nos empregos temporários e aumento nas contratações permanentes, resultando em melhor distribuição das admissões ao longo do ano.

Especificamente no caso da cana-de-açúcar, a mecanização(forte agente de mudança estrutural no mercado de trabalho agropecuário) ocasionará menor uso da mão-de-obra no período da colheita, aumento do desemprego, diminuição dos picos de admissão e consequente alteração na sazonalidade do emprego. Dessa forma, tornam-se necessárias políticas públicas de "re-emprego" no setor canavieiro, como, por exemplo, para qualificar os trabalhadores e reinseri-los em outras funções no setor agropecuário ou até mesmo em outros setores econômicos. 


\section{Referências}

AKAIKE, H. A new Look at the Statistical Model Identification. IEEE Transaction on Automatic Control, AC-19, 716-723. (1974).

ALENCAR, Leonardo S. de. Raízes unitárias e cointegração: uma introdução. Boletim do Banco Central do Brasil, Brasília, p. 171210, abr. 1998.

BAPTISTELLA, C.S.L., VICENTE, M.C.M, FRANCISCO, V.L.F.S, PINO, F.A. O Trabalho Volante na Agricultura Paulista e sua Estacionalidade, 1985-1993. Agricultura em São Paulo, São Paulo, 41(3):61-83,1994.

BOX, George E. P.; JENKINS, Gwilym M.; REINSEL, Gregory C. Time series analysis: forecasting and control. $3^{\text {rd }}$. New Jersey: Prentice Hall, 1994, 598p.

BOX, G.E.P. and PIERCE, D.A. Distribution of residual autocorrelations in autoregressive moving average time series models. Journal of the American Statistical Association, 65, p,1509-26. 1970.

BRASIL. Ministério do Trabalho e Emprego. CADASTRO GERAL DE EMPREGADOS E DESEMPREGADOS (CAGED). Disponível em

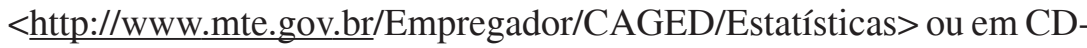
ROM. Acesso em 21/01/2007.

DICKEY, David A.; HASZA, D.P.; FULLER, W.A. Testing for unit roots in seasonal time series. Journal of the American Statistical Association, v.79, p.355-67, 1984.

DICKEY, David A; FULLER, Wayne A. Distribution of the es-timators for autoregressive time series with units root. Journal of the American Statistical Association, Whashington, v.74, n.366, p.427-31, Jun. 1979. 
; L Likelihood ratio statistics for autoregressive time series with a unit root. Econometrica, Chicago, v.49, p 1057-72, Jul. 1981.

FREDO, C.E et al. Cana-de-açúcar - Índice de Mecanização em SP. Agroanalysis, Fundação Getúlio Vargas, 01 mar. 2008

FREITAS, S.M. de; MARGARIDO, Mario A; BARBOSA, Marisa Z.; FRANCA, Teresinha J.F. Análise da Dinâmica de Transmissão de Preços no Mercado Internacional de Farelo de Soja, 1990-99. Agricultura em São Paulo, v.48(1):1-20. 2001.

FREITAS, S.M. de; FERREIRA, C.R.R.P.T.; BARBOSA, M.Z. Oportunidades e entraves à expansão de dendeicultura brasileira. Agricultura em São Paulo, São Paulo, v. 45, t.2, p.1-16, abr. 1998.

GARAY, A. B. S. Reestruturação Produtiva e Desafios de Qualificação: Algumas Considerações Críticas. Disponível em http://www.cefetsp.br/ edu/eso/globalizacao/desafioqualificacao.html. Acessado em 21/10/2008.

GRANGER,C.W.J.; NEWBOLD, P. Spurious regressions in econometrics. Journal of Econometrics, Nottingham, v.2, p. 111-20, Jul. 1974.

LJUNG, G.M. and BOX, G.E.P. On a measure of lack of fit in time series models. Biometrika, 66, 67-72. 1978.

MACKINNON, James G. Critical values for cointegration tests. In: ENGLE, Robert F. ; GRANGER, W. J. Long-run economic relationships: readings in cointegration. New York: Oxford University Press, 1991. p.267-76.

MARGARIDO, Mário A.; ANEFALOS, Lilian C. Testes de raiz unitária e o software SAS. Agricultura em São Paulo, São Paulo, v. 46, t.2, p.19-45, 1999. 
MARGARIDO, Mario A. Transmissão de preços internacionais de suco de laranja para preços ao nível de produtor de laranja no Estado de São Paulo. São Paulo: IEA. 1998. 127p. (Coleção Estudos Agrícolas, 6/98).

MILLS, Terence C. Time series techniques for economists. New York: Cambridge University, 1990.377p.

NELSON, Charles R.; PLOSSER, Charles I. Trends and random walks in macroeconomic time series. Journal of Monetary Economics, v.10, p.139-62, 1982.

Ng S. PERRON P. Unit root test in ARMA models with data-dependent methods for the selection of the truncation lag. Journal of the American Statisitcal Association, v.90, p.268-281. 1995.

PASTORE, Affonso C. A oferta de produtos agrícolas no Brasil. In: PASTORE, José. Agricultura e desenvolvimento. S.n.t., 1973. p.11349.

PASTORE, Affonso C.; ALVES, E.; RIZZIERI, J. A inovação induzida e os limites à modernização na agricultura brasileira. Brasília: EMBRAPA, 1974. (Teoria e Metodologia, v.2).

PHILLIPS, Peter C.B. Understanding spurious regressions in econometrics. Journal of Econometrics, North-Holland, v. 33, n.3, p.311-40, Dec. 1986.

RAO, B. Bhaskara. (Ed.). The econometrics of disequilibrium models. In:__ Cointegration for the applied economist. New York: ST. Martin's Press, 1994. p.1-8.

RAY, Debraj. Development Economics. United States of America: Princeton University Press. 1998. 848p.

REZENDE, Gervásio Castro de. Políticas trabalhista, fundiária e de 
crédito agrícola no Brasil. Rev. Econ. Sociol. Rural, Jan./Mar. 2006, vol.44, no.1, p.47-78. ISSN 0103-2003.

SANTOS, Robério F. dos. Presença de vieses e mudança técnica na agricultura brasileira. São Paulo: IPE/USP. 1986. (Ensaios Econômicos, 63).

SCHWARZ, G. Estimating the dimension of a model. Annals of Statistics 6: 461-464. 1978.

SILVA, J. G. D., KAGEYAMA, A. A., ROMÃO, D. A., WAGNER NETO, J. A., \& PINTO, L. C. G. (1983). Tecnologia e campesinato: O caso brasileiro. Revista de Economia Política, 3(4).

TSUNECHIRO, A. et al., Valor da Produção Agropecuária do Estado de São Paulo 2007. Informações Econômicas, São Paulo, v. 38, no. 4, abr. 2008. p. 78-89.

VANDAELE, Walter. Applied time series and Box-Jenkins models. New York: Academic Press, 1983. 417p.

YAFEE, Robert; McGEE, Monnie. Introduction to time series analysis and forescasting: with applications of SAS and SPSS. United States of America: Academic Press, 2000. 528p. 
Abstract: This paper used the X-12 method, seasonal unit root and Autoregressive Integrated Moving Average Model to identify and to model the generator process of rural employment in the state of São Paulo in the period from January 1996 to December 2006. The results show that there is strong seasonal demand for occasional rural employment from April to August for several main harvests. The seasonal unit root test detected the presence of seasonal unit root. This result confirms the presence of a seasonal pattern in the rural employment time series. The ARIMA model caught the rural employment dynamism for occasional workers. It was necessary to add three moving average parameters of small orders, beyond a seasonal moving average parameter, and an autoregressive parameter which represents the hiring of permanent workers.

Keywords: Rural employment, seasonality, ARIMA model, X-12 method. 